\title{
Host plant preference of Metcalfa pruinosa (Say, 1830) (Hemiptera: Flatidae) in the north of Hungary
}

\author{
András Bozsik \\ University of Debrecen, Institute of Plant Protection, Debrecen, Hungary \\ bozsik@agr.unideb.hu
}

\section{SUMMARY}

Citrus flatid planthopper, a native insect to North America had for a long time a scarce economic importance there. However, being polyphagous made little damage on citrus trees and some ornamentals. In 1979 it was introduced to Italy where it established and spread quickly. It is now an invasive alien species continually spreading in South and Central Europe causing considerable damage in fruit crops and various ornamentals. Present study shows the results of a series of observations carried out from 2011 to 2015 at a number of habitats in north of Hungary. The pest could be found at each habitat but the hedge, the tree row, the gardens and the orchard/vineyard were the most infested. Frequency and population density of Metcalfa pruinosa were considerable on Asteraceae, Cannabaceae, Fabaceae, Juglandaceae, Lamiaceae, Rosaceae and Sapindaceae. Typical vegetation could be functionally classified as ornamental plants, trees/shrubs, fruit plants, weeds and feral plants. Feral plants - some of them also invasive alien species - were found at each habitat. Plant species native to America were among them the most populated. As the hedgerows were neglected, and most gardens, orchards and vineyards abandoned, these are excellent conditions for the quick and long-lasting establishment of the pest as well as they may be reservoirs to infest cultivated fruit crops and ornamentals. The hedgerow was situated along a railway line. The length of similar hedges can be merely in Pest county several hundred km, which means M. pruinosa has plenty of opportunity for spreading along the railway and infest agricultural and ornamental cultures. On the surveyed alfalfa and maize fields, accidentally very few nymphs and adults were observed. Although, the population density of $\mathrm{M}$. pruinosa was considerable on many hostplants, economic damage or yield losses could not be detected. Economic or significant damage was observed only on roses, raspberries and stinging nettle. This later is cultivated in Germany and Finland. The applied horticultural oil was efficient.

Keywords: Metcalfa pruinosa, host plant, hedgerow, tree row, garden, orchard, vineyard

\section{INTRODUCTION}

Citrus flatid planthopper (CFP) Metcalfa pruinosa (Say, 1870) is native to North America from where it was accidentally introduced to Italy in 1979 (Zangheri and Donadini, 1980). After a rapid spreading in Italy it managed to get to more than 15 European countries (Strauss, 2010). Its harm is little in the USA: some fruit trees and ornamentals suffered little damage and aesthetic injury (Mead, 1969). Being polyphagous, M. pruinosa attacked various cultivated and wild trees, shrubs and weedy plants in Italy (Bagnali and Luccchi 2000). Sucking of nymphs can cause deformation and injury of shoots and twigs leading to wilt and destruction. Grape quality damaged considerably as a consequence of nymphs' feeding (acidity and sugar content altered) and also soybean suffered a 30-40\% yield loss in Italy (Ciampolini et al., 1987). Grape quality decreased heavily by the honeydew production of $M$. pruinosa and the following sooty mould formation in France (Della Giustina and Navarra, 1993). Ornamentals in nurseries and parks are in danger because of the waxy filaments produced on leaves and shoots by CFP (Lauterer, 2002; Strauss, 2010). It was revealed that some $M$. pruinosa were infested with various phytoplasmas but they could not transmit them in experiments (Bressan et al., 2006 in Strauss, 2010).

In Europe, M. pruinosa has been settled in Italy, France (Della Giustina, 1986), Slovenia (Sivic, 1991 in Strauss 2010), Switzerland (Jermini et al., 1995), Croatia (Maceljski et al., 1995 in Strauss, 2010), Austria (Kahrer and Moosbeckhofer, 2003), Greece (Drosopoulos et al., 2004 in Strauss 2010), Spain (Pons et al., 2002 in Strauss, 2010), Serbia and Montenegro (Hrncic, 2003), Hungary (Orosz and Dér, 2004), Bulgaria (Tomov et al., 2006 in Strauss, 2010), Turkey (Karsavuran and Güçlu, 2004 in Strauss, 2010), Bosnia Herzegovina (Gotlin Culjak et al., 2007 in Strauss, 2010), Romania (Grozea et al., 2011) and was found also in Albania, Slovakia and Russia (DAISIE website, 2015). M. pruinosa populations found in the UK and Bohemia were successfully eradicated by insecticide treatments (C. Malumphy and P. Lauterer, personnel communication in Strauss, 2009).

In Bohemia, Austria and Romania started thorough observations and investigations to get to know its spread, host plants and control. In Austria using the CLIMEX ${ }^{\circledR}$ programme various parameters (temperature index, diapausa index, moisture index, cold, wet, dry and heat stresses) were investigated in order to find the susceptible Austrian areas and cultures and also worked on the control opportunities (Strauss, 2010).

M. pruinosa occurred in Hungary (Budapest) in 2004 but its expansion and injury have not been reported considerable. The pest was observed on feeding Ambrosia artemisiifolia L. in Szabolcs-Szathmár county (Lajos Szőke personnel communication, 2011), which was considered as a possible natural weed control.

The aim of this study was studying the most frequent host plants of $M$. pruinosa monitored in various habitats of Gödöllő and countryside as well as to conclude with assessing the population density to the preference of the pest. 


\section{Morphology}

Mead (1969) and Lauterer (2002) gave a detailed morphological characterisation on adults and nymphs, thus here only a very brief description will be provided. Adults are 7-8 $\mathrm{mm}$ in length. Dorsal surface of the body and forewings are blackish brown. Body and forewings are covered with a whitish powdery secretion making the blackish colour grey-bluish. The nymphs' body is flattened and white, covered with a dense waxy substance forming long filaments at the apex. The same waxy secretion is produced on leaves and shoots where the nymphs feed. The $5^{\text {th }}$ - last one - instar is 5-6 mm long. Development stage of nymphs can be distinguished by the size of head capsule and wing pads (Lauterer, 2002).

\section{Life cycle}

M. pruinosa has one generation a year and overwinters as eggs laid in the bark of damaged twigs (Mead, 1969, Lauterer 2002). In France and Austria, nymphs can hatch from May to mid July and suck phloem sup of host plants and produce a lot of honeydew. They have five growth stages. Finishing development adults emerge in August and begin laying eggs. Egg production can be maximum 90 eggs (Della Giustina, 1987; Kahrer et al., 2009).

\section{Host plants}

M. pruinosa is a polyphagous planthopper feeding on a great diversity of plants. Unfortunately, because of this high diversity it is difficult to prove its preference and future injury.

In the USA, CFP was found on citrus, grape fruit, orange, grape, many forest and fruit trees, shrubs and some herbs (Mead, 1969). Bagnoli and Lucchi (2000) reported more than 200 host plants from different families for M. pruinosa in Italy. In the Czech Republic, M. pruinosa slightly damaged ornamental plants like Thuja occidentalis L., Juniperus communis L., Sorbus aucuparia L., Lilium sp. and were found also on some woody species (Lauterer, 2002). In Austria (Vienna), the following host plant genera were observed: Acer, Aesculus, Ailanthus, Amaranthus, Amelanchier, Amorpha, Arctium, Aronia, Artemisia, Aucuba, Ballota, Bryonia, Buddleja, Buxus, Calycanthus, Campanula, Canna, Carpinus, Catalpa, Ceratostigma, Cercis, Chaenomeles, Chelidonium, Chenopodium, Clematis, Clivia, Convolvulus, Conyza, Cornus, Corylus, Cotinus, Cotoneaster, Crataegus, Cucurbita, Daphne, Daucus, Deutzia, Dipsacus, Duchesnea, Echium, Epilobium, Epimedium, Erigeron, Euonymus, Fagus, Falcaria, Fallopia, Ficus, Fontanesia, Forsythia, Fraxinus, Galium, Geranium, Geum, Glechoma, Hedera, Heptacodium, Heracleum, Hibiscus, Hippophae, Humulus, Hydrangea, Hypericum, Jasminum, Juglans, Knautia, Koelreuteria, Kolkwitzia, Laburnum, Lamium, Leonurus, Ligustrum, Lonicera, Lycium, Lythrum, Magnolia, Mahonia, Majorana, Malus, Malva, Medicago, Mercurialis, Mespilus, Morus, Nerium, Oxalis, Parthenocissus, Paulownia, Pennisetum, Petroselinum, Phaseolus, Philadelphus, Physocarpus, Phytolacca, Pieris, Pinus, Plantago, Platanus, Polygonum, Poncirus, Populus, Potentilla, Prunus, Ptelea, Pyracantha, Pyrus, Quercus, Rhododendron, Rhus, Ribes, Robinia, Rosa, Rosmarinus, Rubus, Rumex, Salix, Sambucus, Silene, Sisymbrium, Skimmia, Solanum, Solidago, Sonchus, Sorbus, Spartium, Spiraea, Staphylea, Symphoricarpus, Syringa, Tagetes, Tanacetum, Taraxacum, Taxus, Thuja, Tilia, Triticum, Ulmus, Urtica, Vaccinium, Veronica, Viburnum, Vinca, Viola, Vitis, Weigela, Wisteria, Yucca. Nymphs, adults or both of them were found on these genera (Kahrer et al., 2009). There was presented another study indicating that 251 plant species among them numerous horticultural and agricultural varieties were attacked either by the nymphs or imagines of the planthopper (Moosbeckhofer et al., 2009). In Serbia (Belgrad), M. pruinosa were reported on woody species in the genera: Acer, Aesculus, Gleditchia, Robinia, Ailanthus, Populus, Platanus, Prunus, Pyrus, Ulmus, Tilia, Cornus, Fraxinus, Quercus and Thuja (Mihajlović, 2007). In Romania, it was collected and seen on Acer saccharinum L., Juglans nigra L., Juniperus sp., Thuja occidentalis L., Buxus sempervirens L., Albizia julibrissin Durazz., Potentilla (Dasiphora) fruticosa L., Cycas revoluta Thunb., Vitis vinifera L., Atriplex hortensis L., Sambucus nigra L., Melissa officinalis L., Philadelphus coronaries L., Ligustrum vulgare L., Hibiscus rosa-sinensis l. and Rosa sp. (Grozea et al., 2011). In western counties of Romania M. pruinosa nymphs were observed on 45 host plants species at various habitats like parks, orchard and vineyard. The most important plants were Acer negundo L., Acer pseudoplatanus L., Acer campestre L., Acer platanoides L., Catalpa bignonioides Walter, Hibiscus syriacus L., Juglans regia L., Ligustrum vulgare, Malus domestica L., Prunus persica (L.) Batsch, Prunus armeniaca L., Prunus domestica L., Tilia cordata Mill., and Vitis vinifera L. In Greece, 26 ornamental, 15 fruit, nine weed and two vegetable plant species were reported as associated with CFP. Among them there were three monocotyledonous species, each of them weeds such as Bromus sp., Digitaria sanguinals (L.) Scop. and Setaria sp. (Souliotis et al., 2008).

In Hungary, M. pruinosa was observed in Budapest on the following plants: Acer sp., Aesculus hippocastanum L., Berberis sp., Crataegus sp., Hibiscus sp., Syringa sp., Ulmus sp. (Orosz and Dér, 2004). In addition, Bozsik (2012) listed some plants on which adults and waxy secretion of $M$. pruinosa were observed: Acer negundo L., Celtis occidentalis L., Clematis vitalba L., Crataegus monogyna Jacq., Lycium halimifolium L., Morus alba L., Prunus padus L., Prunus serotina Ehrh., Prunus spinosa L., Robinia pseudoacacia L., Rosa canina L., Ulmus campestris L. and Vitis vinifera L. 


\section{Control of Metcalfa pruinosa}

In its native area usually there is no need for control except in case of obvious damage which is a rarity (Mead, 1969). Cutting twigs infested with eggs or treatments with horticultural oil and insecticidal soap is enough against M. pruinosa (Rebek, 2009). Chlorpyriphos and imidacloprid was efficient in Austria (Kahrer and Moosbeckhofer, 2003). Fenitrothion was used successfully in the Czech Republic (Lauterer, 2002). Strauss (2009) studied control opportunities with special regard for the use of the natural enemy, Neodryinus typhlocybae (Ashmead, 1893) (Hymenoptera: Dryinidae) which has been released in Italy, France and Slovenia. She showed that $N$. typhlocybae attacked and parasitised only M. pruinosa and the native European planthoppers were saved (Strauss, 2009). Malausa et al. (2003) introduced N. typhlucybae in the south of France in 1996 to control M. pruinusa. The parasitoid was released in about sixty sites and after five years of the first introduction the authors evaluated the establishment and dispersal of $N$. typhlucybae. They found established populations in $51(86 \%)$ sites.

\section{Route of spreading}

Spreading of M. pruinosa in Hungary could be with transported tree and ornamental seedlings from areas where the pest is established or the planthopper itself could migrate from the same localities. According to Lauterer (2002) the natural annual spreading of $M$. pruinosa is about $50 \mathrm{~m}$ on each direction. Grozea et al. (2011) thought $M$. pruinosa individuals flew in Romania (Temes County) from the neighbouring Serbia and Hungary. It means that the density of this planthopper in Hungary was estimated as high.

\section{MATERIALS AND METHODS}

The surveyed sites were found at Gödöllő and Máriabesnyő about $25 \mathrm{~km}$ from Budapest. Most areas at Máriabesnyő belonged to the Szent István University and localised along the railway running towards Budapest. Localities at Gödöllö were in the centre of the town and at the Blaha district. The Blaha district is an area where orchards, vineyards and vegetable gardens were found (Table 1).

Hedge (Máriabesnyő)

Vegetation of the area consisted of European and some adventive (with American and Asian origin) trees and bushes that created a very dense structure.

Tree row (Máriabesnyő)

A line of trees - along an alfalfa field and a field path - consisted mainly of matured trees and some saplings. The trees generally do not meet each other.

Stinging nettle spot (Máriabesnyő)

It was found at the border of a deciduous wood below the tree branches.

Backside garden (Máriabesnyő)

It contained ornamental plants, fruit trees and shrubs.

Maize field (Máriabesnyő)

The field was overgrown with weeds such as Artemisia vulgaris, Ambrosia artemisiifolia, Cannabis sativa spontanea, Chenopodium album and Atriplex tatarica. The weeds hampered even the moving in the field. Four times 25 corn plants were visually surveyed along a transect line. No pesticides were applied.

Alfalfa field (Máriabesnyö)

The field was overrun with weeds such as Rumex obtusifolius, Agropyron repens, Setaria glauca, Senecio vulgaris and Lactuca serriola. The alfalfa stand was thinned out and the weeds covered more space than the alfalfa. Captures were obtained by sweeping net ( 4 x 25 sweeps). The individuals captured by sweeping were taken into a freezer, then dried for a while and identified immediately. At the field no pesticides were applied.

Backside garden (Gödöllö)

It contained ornamental and fruit trees and shrubs.

Orchard and vineyard (Gödöllö)

The site was grown over with fruit trees and grapevine.

In the hedge, five shoots of randomly selected trees or bushes - or weedy plants - were observed from a 50 $\mathrm{cm}$ distance and the number of nymphs and adults of $M$. pruinosa was recorded (abbreviated as VO1). In case of the other habitats (except the alfalfa field), plants were examined similarly but instead of counting the pest 
individuals the following approximation was used: 1 = weak infestation ( $<5$ nymphs or adults on a shoot part); 2 $=$ middle infestation (5 - 10 nymphs or adults on a shoot part); 3 = strong infestation ( $>10$ nymphs or adults on a shoot part; abbreviated as VO2) (Kahrer et al., 2009).

Also the efficiency of chemical control administered at the Máriabesnyő garden was approximately assessed. A horticultural oil (Vectaphid A EC: 83\% paraffin oil +17\% Atplus 309 F - in a concentration of 0.5\%) was applied two times in July and once in August in 2015 with a manual backpack sprayer.

Basic data of the surveys

\begin{tabular}{|c|c|c|c|c|}
\hline Site and date & $\begin{array}{l}\text { Geographic } \\
\text { position Altitude }\end{array}$ & Habitat Size & $\begin{array}{l}\text { Surveying } \\
\text { method }\end{array}$ & $\begin{array}{l}\text { Surveying } \\
\text { frequency }\end{array}$ \\
\hline $\begin{array}{l}\text { Máriabesnyő } 2013 \\
\text { from early July to } \\
\text { early October }\end{array}$ & $\begin{array}{l}47^{\circ} 36^{\prime} 3^{\prime \prime} \mathrm{N} \\
19^{\circ} 21 \text { '59"E } 209 \mathrm{~m}\end{array}$ & hedge $660 \mathrm{~m}$ & $\begin{array}{l}\text { visual } \\
\text { observation = } \\
\text { VO1 }\end{array}$ & weekly \\
\hline $\begin{array}{l}\text { Máriabesnyő } 2015 \\
\text { from early July to } \\
\text { early September }\end{array}$ & $\begin{array}{l}47^{\circ} 35^{\prime} 54^{\prime \prime} \mathrm{N} \\
19^{\circ} 22^{\prime} 56^{\prime \prime} \mathrm{E} \\
211 \mathrm{~m}\end{array}$ & $\begin{array}{l}\text { tree row } \\
649 \mathrm{~m}\end{array}$ & VO2 & weekly \\
\hline $\begin{array}{l}\text { Máriabesnyő 2013-15 } \\
\text { from early June to } \\
\text { early October }\end{array}$ & $\begin{array}{l}47^{\circ} 35^{\prime} 37^{\prime \prime} \mathrm{N} \\
19^{\circ} 22^{\prime} 59^{\prime \prime} \mathrm{E} \\
196 \mathrm{~m}\end{array}$ & $\begin{array}{l}\text { stinging } \\
\text { nettle spot } \\
60 \mathrm{~m} 2 \\
\end{array}$ & VO2 & weekly \\
\hline $\begin{array}{l}\text { Máriabesnyő 2011-15 } \\
\text { from early April to } \\
\text { early October }\end{array}$ & $\begin{array}{l}47^{\circ} 35^{\prime} 44^{\prime \prime} \mathrm{N} \\
19^{\circ} 22^{\prime} 54^{\prime \prime} \mathrm{E} \\
204 \mathrm{~m}\end{array}$ & $\begin{array}{l}\text { backside } \\
\text { garden } \\
680 \mathrm{~m} 2 \\
\end{array}$ & VO2 & weekly \\
\hline $\begin{array}{l}\text { Máriabesnyő } 2015 \\
\text { from early July to } \\
\text { early September }\end{array}$ & $\begin{array}{l}47^{\circ} 35^{\prime} 51^{\prime \prime} \mathrm{N} \\
19^{\circ} 22^{\prime} 29^{\prime \prime} \mathrm{E} \\
207 \mathrm{~m}\end{array}$ & $\begin{array}{l}\text { maize field } \\
2.7 \text { ha }\end{array}$ & VO1 & weekly \\
\hline $\begin{array}{l}\text { Máriabesnyő } 2015 \\
\text { from early July to } \\
\text { early September }\end{array}$ & $\begin{array}{l}47^{\circ} 35^{\prime} 52^{\prime \prime} \mathrm{N} \\
19^{\circ} 22^{\prime} 5^{\prime \prime} \mathrm{E} \\
212 \mathrm{~m}\end{array}$ & $\begin{array}{l}\text { alfalfa field } \\
7.7 \text { ha }\end{array}$ & sweep netting & weekly \\
\hline $\begin{array}{l}\text { Gödöllö 2011-15 } \\
\text { from early April to } \\
\text { early October }\end{array}$ & $\begin{array}{l}47^{\circ} 36^{\prime} 9^{\prime \prime} \mathrm{N} \\
19^{\circ} 21^{\prime} 27^{\prime \prime} \mathrm{E} \\
188 \mathrm{~m}\end{array}$ & $\begin{array}{l}\text { backside } \\
\text { garden } \\
1296 \mathrm{~m} 2 \\
\end{array}$ & VO2 & weekly \\
\hline $\begin{array}{l}\text { Gödöllö 2011-15 } \\
\text { from early April to } \\
\text { early October }\end{array}$ & $\begin{array}{l}47^{\circ} 37^{\prime} 25^{\prime \prime} \mathrm{N} \\
19^{\circ} 19^{\prime} 56^{\prime \prime} \mathrm{E} \\
192 \mathrm{~m}\end{array}$ & $\begin{array}{l}\text { orchard and } \\
\text { vineyard } \\
2160 \mathrm{~m} 2 \\
\end{array}$ & VO2 & weekly \\
\hline
\end{tabular}

\section{RESULTS}

Most important habitats in North America of CFP are mixed deciduous woods and open areas overgrown with bushes. Also in Italy, the pest started its spread from an open bushland (Moosbeckhofer et al., 2009). In Central and South Europe, similar habitats were sampled. Regarding the Czech Republic, it was found in a nursery of ornamentals (Lauterer, 2002) and in Austria, the pest was first found and sampled in gardens, parks, a city wood with ornamental and not cultivated trees and shrubs and a cemetery (Moosbeckhofer et al., 2009).

In Romania, similarly, parks, green areas, public gardens, an orchard, a vineyard and vegetation along the roads were observed and surveyed (Preda and Skolka, 2011; Grozea et al., 2015). In Greece, citrus and olive groves were investigated to monitor CFP (Souliotis et al., 2008). In the present study, a hedge, a tree row and various horticultural and agricultural spaces such as gardens, an orchard, a vineyard, two fields and a nettle stand were examined.

M. pruinosa individuals or their waxy secretion were observed on 57 species of 31 plant families at seven survey sites (Table 2). The most objective investigation was carried out at the hedge where 23 species of 14 families were associated with $M$. pruinosa. $R$. pseudoacacia, $P$. spinosa, C. vitalba, U. campestris, $P$. cerasifera were the most attacked host plants (Table 3). At the tree row the pest was observed on 12 plants of eight families and R. pseudoacacia, C. occidentalis and A. pseudoplatanus showed the highest pest density (Table 4). The vegetation of the backside garden at Máriabesnyő indicated the highest number of infested host plants. R. idaeus, $C$. occidentalis, $H$. lupulus, $R$. damascena, $J$. regia, $R$. canina and $H$. helix revealed a strong $M$. pruinosa population (Table 5). In the Gödöllö backside garden with 15 infested host plants C. occidentalis, J. regia and $R$. damascena were the most preferred plants (Table 6). In the Gödöllö orchard eight plants was infested and the pest was observed mainly on $C$. occidentalis, $R$. pseudoacacia and A. negundo (Table 7). Plants of the stinging 
Host plants associated with Metcalfa pruinosa at Gödöllö and environment $(\mathrm{H}=$ hedge, $\mathrm{T}=$ tree row, $\mathrm{BB}=$ backside garden Máriabesnyő, BG = Backside garden Gödöllö, $\mathbf{O G}=$ orchard, vineyard Gödöllö)

\begin{tabular}{|c|c|c|c|c|c|}
\hline Host plant & H & $\mathbf{T}$ & BM & BG & OG \\
\hline Acer campestre & + & + & + & + & \\
\hline Acer negundo & + & & & + & + \\
\hline Acer platanoides & + & + & & & \\
\hline Acer pseudoplatanus & & + & & & \\
\hline Achillea colinna & & & + & & \\
\hline Alcea rosea & & & + & & \\
\hline \multicolumn{6}{|l|}{ Ailanthus altissima } \\
\hline Ambrosia artemisiifolia & + & & & & \\
\hline Buxus sempervirens & & & & + & \\
\hline $\begin{array}{l}\text { Caryopteris incana } x \\
\text { Caryopteris mongholica }\end{array}$ & & & + & & \\
\hline Castanea sativa & & & + & & \\
\hline Celtis occidentalis & + & + & + & + & + \\
\hline Chrysanthemum indicum & & & + & & \\
\hline Clematis vitalba & + & & & & \\
\hline Cornus sanguinea & & + & + & & \\
\hline Cosmos bipannatus & & & + & & \\
\hline Crataegus monogyna & + & & + & & \\
\hline Euonymus europeus & + & & + & & \\
\hline Euonymus japonicus & & & + & & \\
\hline Euphorbia salicifolia & + & & & & \\
\hline Forsythia suspensa & & & & + & \\
\hline Fragaria vesca & + & & & & \\
\hline Fraxinus ornus & & + & & & \\
\hline Gleditsia triacanthos & & & & + & \\
\hline Hedera helix & & & + & + & \\
\hline Helianthus tuberosus & & & + & & \\
\hline Humulus lupulus & + & & + & & + \\
\hline Juglans regia & + & + & + & + & + \\
\hline Mahonia aquifolium & & & & + & \\
\hline Malus domestica & + & + & + & & \\
\hline Morus nigra & + & & & & \\
\hline Nerium oleander & & & + & & \\
\hline Populus nigra & & + & & & \\
\hline Prunus cerasifera & + & & & & \\
\hline Prunus domestica & & & + & + & \\
\hline Prunus serotina & + & + & & & \\
\hline Prunus spinosa & + & & & & \\
\hline Philadelphus coronarius & & & & + & \\
\hline Physostegia virginiana & & & + & & \\
\hline Robinia pseudoacacia & + & + & & & + \\
\hline Rosa canina & + & + & + & + & + \\
\hline Rosa damascena & & & + & + & \\
\hline Rubus fruticosus & & & + & & \\
\hline Rubus idaeus & & & + & & \\
\hline Salvia sclarea & & & + & & \\
\hline Sambucus nigra & + & & & & \\
\hline Solanum nigrum & + & & & + & \\
\hline Solidago canadensis & & & + & & + \\
\hline Spirea $x$ vanhouttei & & & & + & \\
\hline Syringa vulgaris & & & + & & \\
\hline Ulmus campestris & + & & & & \\
\hline Urtica urens & & & + & & \\
\hline Vitis vinifera & & & + & & + \\
\hline Weigela florida & & & + & & \\
\hline
\end{tabular}


Host plants and frequency of Metcalfa pruinosa (hedge, Máriabesnyö, 2013, + = waxy secretion found)

\begin{tabular}{|l|l|l|l|l|}
\hline \multirow{2}{*}{ Plant } & \multirow{2}{*}{ Number of shoots } & \multicolumn{3}{|c|}{ Number of M. pruinosa } \\
\cline { 2 - 5 } & & nymphs & adults & waxy rests \\
\hline Acer campestre & 9 & - & 5 & + \\
\hline Acer negundo & 13 & - & 40 & + \\
\hline Acer platanoides & 35 & 2 & 41 & + \\
\hline Ailanthus altissima & 2 & 2 & 2 & + \\
\hline Ambrosia artemisiifolia & 1 & - & - & + \\
\hline Celtis occidentalis & 11 & 2 & 4 & + \\
\hline Clematis vitalba & 99 & 169 & 36 & + \\
\hline Crataegus monogyna & 56 & 4 & 23 & + \\
\hline Euonymus europeus & 8 & - & 10 & + \\
\hline Euphorbia salicifolia & 1 & - & - & + \\
\hline Fragaria vesca & 1 & - & 1 & + \\
\hline Humulus lupulus & 10 & - & 13 & + \\
\hline Juglans regia & 2 & - & 4 & + \\
\hline Malus domestica & 3 & 18 & - & + \\
\hline Morus nigra & 1 & - & - & + \\
\hline Prunus cerasifera & 60 & 39 & 66 & + \\
\hline Prunus serotina & 11 & - & 5 & + \\
\hline Prunus spinosa & 84 & 251 & 36 & + \\
\hline Robinia pseudo-acacia & 317 & 1160 & 101 & + \\
\hline Rosa canina & 6 & - & 3 & + \\
\hline Sambucus nigra & 1 & - & - & + \\
\hline Solanum nigrum & 1 & - & - & + \\
\hline Ulmus campestris & 130 & 114 & 71 & + \\
\hline
\end{tabular}

Host plants and frequency of Metcalfa pruinosa (tree row, Máriabesnyő, 2015)

\begin{tabular}{|l|l|l|l|}
\hline \multirow{2}{*}{ Plant } & \multicolumn{3}{|l|}{ M. pruinosa } \\
\cline { 2 - 4 } & nymphs & adults & waxy filaments \\
\hline Acer campestre & - & 1 & - \\
\hline Acer platanoides & - & 1 & - \\
\hline Acer pseudoplatanus & 1 & 2 & + \\
\hline Celtis occidentalis & 2 & 2 & + \\
\hline Cornus sanguinea & - & 1 & + \\
\hline Fraxinus ornus & 1 & 1 & + \\
\hline Juglans regia & 1 & 1 & + \\
\hline Malus domestica & - & 1 & - \\
\hline Populus nigra & - & 1 & + \\
\hline Prunus serotina & - & 1 & - \\
\hline Robinia pseudoacacia & 1 & 2 & + \\
\hline Rosa canina & - & 1 & - \\
\hline
\end{tabular}

nettle stand were strongly infested. The average number of nymphs and adults of $M$. pruinosa varied between 30 and 100 individuals or more.

Tables 8-12 specify the host plant families classified in functional groups like ornamental plants, trees, fruit plants, weeds and feral plants. The plants studied were almost exclusively dicotyledonous species except maize. CFP was found also on maize plants. Their density was very low: some $4^{\text {th }}, 5^{\text {th }}$ instar nymphs and adults of $M$. pruinosa as well as some waxy secretion were observed on the leaves and stalks. The infestation on maize can be regarded as very scarce. The population density of the pest in the alfalfa field was accidental. At this field along the tree row investigated, altogether two adults were found in the net during the survey period in the late of August. On the alfalfa plants no nymphs or waxy rests were observed.

The three times applied horticultural oil at the Máriabesnyő garden allowed to decrease the planthopper's density to a tolerable level in roses and raspberries. 
Host plants and frequency of Metcalfa pruinosa (backside garden, Máriabesnyő, 2011-15)

\begin{tabular}{|l|l|l|l|}
\hline \multirow{2}{*}{ Plant } & \multicolumn{3}{|c|}{ M. pruinosa } \\
\cline { 2 - 4 } & nymphs & adults & waxy filaments \\
\hline Acer campestre & 1 & 2 & + \\
\hline Achillea colinna & - & 1 & - \\
\hline Alcea rosea & & 1 & - \\
\hline $\begin{array}{l}\text { Caryopteris incana } \mathrm{x} \\
\text { Caryopteris mongholica }\end{array}$ & - & 1 & + \\
\hline Castanea sativa & 1 & 1 & + \\
\hline Celtis occidentalis & 2 & 2 & + \\
\hline Chrysanthemum & & 1 & + \\
\hline Cornus sanguinea & 1 & 2 & + \\
\hline Cosmos bipinnatus & - & 1 & - \\
\hline Crataegus monogyna & 1 & 1 & \\
\hline Euonnymus europeus & 1 & 1 & + \\
\hline Euonymus japonicus & 1 & 1 & + \\
\hline Hedera helix & 1 & 3 & + \\
\hline Helianthus tuberosus & - & 1 & - \\
\hline Humulus lupulus & 2 & 3 & + \\
\hline Juglans regia & 2 & 2 & + \\
\hline Malus domestica & 1 & 1 & + \\
\hline Nerium oleander & 1 & 1 & + \\
\hline Prunus domestica & - & 1 & + \\
\hline Physostegia virginiana & - & 1 & - \\
\hline Rosa canina & 1 & 2 & + \\
\hline Rosa damascena & 2 & 3 & + \\
\hline Rubus caesius & - & 1 & - \\
\hline Rubus idaeus & 3 & 3 & + \\
\hline Salvia sclarea & - & 2 & + \\
\hline Solidago canadensis & - & 1 & - \\
\hline Syringa vulgaris & - & 1 & - \\
\hline Urtica urens & - & 1 & + \\
\hline Vitis vinifera & 1 & 2 & + \\
\hline Weigela florida & - & 1 & - \\
\hline & & & \\
\hline
\end{tabular}

Host plants and frequency of Metcalfa pruinosa (backside garden, Gödöllö, 2011-15)

\begin{tabular}{|l|l|l|l|}
\hline \multirow{2}{*}{ Plant } & \multicolumn{3}{|c|}{ M. pruinosa } \\
\cline { 2 - 4 } & nymphs & adults & waxy filaments \\
\hline Acer campestre & 1 & 1 & + \\
\hline Acer negundo & 1 & 1 & + \\
\hline Buxus sempervirens & 1 & 1 & + \\
\hline Celtis occidentalis & 2 & 3 & + \\
\hline Forsythia suspensa & - & 1 & - \\
\hline Gleditsia triacanthos & - & 1 & + \\
\hline Hedera helix & - & 1 & - \\
\hline Juglans regia & 2 & 2 & + \\
\hline Mahonia aquifolium & - & 1 & + \\
\hline Philadelphus coronarius & - & 1 & + \\
\hline Prunus domestica & 1 & 1 & + \\
\hline Rosa canina & 1 & 1 & + \\
\hline Rosa damascena & 1 & 2 & + \\
\hline Solanum nigrum & - & 1 & - \\
\hline Spirea $\times$ vanhouttei & - & 1 & - \\
\hline
\end{tabular}


Host plants and frequency of Metcalfa pruinosa (orchard and vineyard, Gödöllö, 2011-15)

\begin{tabular}{|l|l|l|l|}
\hline \multirow{2}{*}{ Plant } & \multicolumn{3}{|c|}{ M. pruinosa } \\
\cline { 2 - 4 } & nymphs & adults & waxy filaments \\
\hline Acer negundo & 1 & 2 & + \\
\hline Celtis occidentalis & 2 & 2 & + \\
\hline Humulus lupulus & 1 & 1 & + \\
\hline Juglans regia & 1 & 1 & + \\
\hline Robinia pseudoacacia & 1 & 2 & + \\
\hline Rosa canina & - & 1 & - \\
\hline Solidago gigantea & - & 1 & + \\
\hline Vitis vinifera & - & 1 & + \\
\hline
\end{tabular}

Functionally classified host plant families (hedge, Máriabesnyő, 2013)

\begin{tabular}{|l|l|l|l|l|l|}
\hline $\begin{array}{l}\text { Plant } \\
\text { family }\end{array}$ & $\begin{array}{l}\text { Trees } \\
\text { shrubs } \\
\text { climbers }\end{array}$ & $\begin{array}{l}\text { Fruit } \\
\text { plants }\end{array}$ & Weeds & $\begin{array}{l}\text { Feral } \\
\text { plants }\end{array}$ & $\begin{array}{l}\text { Total } \\
\text { number of } \\
\text { species }\end{array}$ \\
\hline Adoxaceae & 1 & & & & 1 \\
\hline Asteraceae & & & 1 & & 1 \\
\hline Cannabaceae & 2 & & & & 2 \\
\hline Celastraceae & 1 & & & & 1 \\
\hline Euphorbiaceae & & & 1 & & 1 \\
\hline Fabaceae & 1 & & & & 1 \\
\hline Juglandaceae & & 1 & & & 1 \\
\hline Moraceae & & & & 1 & 1 \\
\hline Ranunculaceae & 1 & & & & 1 \\
\hline Rosaceae & 4 & 2 & & 1 & 7 \\
\hline Sapindaceae & 2 & & & 1 & 3 \\
\hline Simaroubaceae & & & & 1 & 1 \\
\hline Solanaceae & & & 1 & & 1 \\
\hline Ulmaceae & 1 & & & & 1 \\
\hline Number of species & 13 & 3 & 3 & 4 & 23 \\
\hline
\end{tabular}

Functionally classified host plant families (tree row, Máriabesnyő, 2013)

\begin{tabular}{|l|l|l|l|l|}
\hline $\begin{array}{l}\text { Plant } \\
\text { family }\end{array}$ & $\begin{array}{l}\text { Trees } \\
\text { shrubs } \\
\text { climbers }\end{array}$ & $\begin{array}{l}\text { Fruit } \\
\text { plants }\end{array}$ & $\begin{array}{l}\text { Feral } \\
\text { plants }\end{array}$ & $\begin{array}{l}\text { Total } \\
\text { number of } \\
\text { species }\end{array}$ \\
\hline Cannabaceae & 1 & & & 1 \\
\hline Cornaceae & 1 & & & 1 \\
\hline Fabaceae & 1 & & & 1 \\
\hline Juglandaceae & & 1 & & 1 \\
\hline Oleaceae & 1 & & & 1 \\
\hline Rosaceae & 1 & 1 & 1 & 3 \\
\hline Salicaceae & 1 & & & 1 \\
\hline Sapindaceae & 3 & & & 3 \\
\hline Number of species & 9 & 2 & 1 & 12 \\
\hline
\end{tabular}


Functionally classified host plant families (backside garden, Máriabesnyő, 2011-15)

\begin{tabular}{|l|l|l|l|l|l|l|}
\hline $\begin{array}{l}\text { Plant } \\
\text { family }\end{array}$ & $\begin{array}{l}\text { Ornamental } \\
\text { plants }\end{array}$ & $\begin{array}{l}\text { Trees } \\
\text { shrubs } \\
\text { climbers }\end{array}$ & $\begin{array}{l}\text { Fruit } \\
\text { plants }\end{array}$ & Weeds & $\begin{array}{l}\text { Feral } \\
\text { plants }\end{array}$ & $\begin{array}{l}\text { Total } \\
\text { number of } \\
\text { species }\end{array}$ \\
\hline Araliaceae & & 1 & & & & 1 \\
\hline Apocynaceae & 1 & & & & & 1 \\
\hline Asteraceae & 2 & & & 1 & 2 & 5 \\
\hline Cannabaceae & & 2 & & & & 2 \\
\hline Caprifoliaceae & 1 & & & & & 1 \\
\hline Celastraceae & 1 & 1 & & & & 2 \\
\hline Cornaceae & & 1 & & & & 1 \\
\hline Fagaceae & & & 1 & & & 1 \\
\hline Juglandaceae & & & 1 & & & 1 \\
\hline Lamiaceae & 3 & & & & & 3 \\
\hline Malvaceae & 1 & & & & & 1 \\
\hline Oleaceae & 1 & & & & & 1 \\
\hline Rosaceae & 1 & 3 & 3 & & & 7 \\
\hline Sapindaceae & & 1 & & & & 1 \\
\hline Urticaceae & & & & 1 & & 1 \\
\hline Vitaceae & & & 1 & & & 1 \\
\hline Number of species & 11 & 9 & 6 & 2 & 2 & 30 \\
\hline
\end{tabular}

Functionally classified host plant families (backside garden, Gödöllö, 2011-15)

\begin{tabular}{|l|l|l|l|l|l|}
\hline $\begin{array}{l}\text { Plant } \\
\text { family }\end{array}$ & $\begin{array}{l}\text { Ornamental } \\
\text { plants }\end{array}$ & $\begin{array}{l}\text { Trees } \\
\text { shrubs } \\
\text { climbers }\end{array}$ & $\begin{array}{l}\text { Fruit } \\
\text { plants }\end{array}$ & Weeds & $\begin{array}{l}\text { Total } \\
\text { number of } \\
\text { species }\end{array}$ \\
\hline Araliaceae & & 1 & & & 1 \\
\hline Berberidaceae & 1 & & & & 1 \\
\hline Buxaceae & 1 & & & & 1 \\
\hline Cannabaceae & & 1 & & & 1 \\
\hline Fabaceae & 1 & & & & 1 \\
\hline Hydrangeaceae & 1 & & & & 1 \\
\hline Juglandaceae & & & 1 & & 1 \\
\hline Oleaceae & 1 & & & & 1 \\
\hline Rosaceae & 2 & 1 & 1 & & 4 \\
\hline Sapindaceae & & 1 & & 1 & 2 \\
\hline Solanaceae & & & & 1 & 1 \\
\hline Number of species & 7 & 4 & 2 & 2 & 15 \\
\hline
\end{tabular}

Functionally classified host plant families (orchard and vineyard, Gödöllö, 2011-15)

\begin{tabular}{|l|l|l|l|l|}
\hline $\begin{array}{l}\text { Plant } \\
\text { family }\end{array}$ & $\begin{array}{l}\text { Trees } \\
\text { shrubs } \\
\text { climbers }\end{array}$ & $\begin{array}{l}\text { Fruit } \\
\text { plants }\end{array}$ & Feral plants & $\begin{array}{l}\text { Total } \\
\text { number of } \\
\text { species }\end{array}$ \\
\hline Asteraceae & & & 1 & 1 \\
\hline Cannabaceae & 2 & & & 2 \\
\hline Fabaceae & 1 & & & 1 \\
\hline Juglandaceae & & 1 & & 1 \\
\hline Rosaceae & 1 & & & 1 \\
\hline Sapindaceae & & & 1 & 1 \\
\hline Vitaceae & & 1 & & 1 \\
\hline Number of species & 5 & 2 & 2 & 8 \\
\hline
\end{tabular}




\section{DISCUSSION}

M. pruinosa is a polyphagous pest preferring saplings and shoots of trees and bushes but it can feed also on weed plants. It attacks mainly dicotyledonous plants but some studies reported their feeding on monocotyledonous vegetation. In Greece, it was stated on Bromus sp., D. sanguinalis and Setaria sp. (Souliotis et al., 2008) and in Austria on winter wheat, Canna indica and not determined grasses (Moosbeckhofer et al., 2009). There are many data on its host plants and most of them are ornamental and fruit trees/shrubs and also some horticultural or agricultural crops.

Regarding the Central European region, Austrian data are the most detailed and also some Romanian papers can be of importance. Moosbeckhofer et al. (2009) published a thorough study in which they listed 251 plant species observed and evaluated in Vienna and its countryside with additional information on the degree of infestation of the planthopper. This assessment is the same which was presented in the methodical part and abbreviated as OV2. Except 16 species (A. rosea, A. artemisiifolia, C. incana, C. sativa, C. occidentalis, Ch. indicum, C. bipennatus, E. salicifolia, F. vesca, G. triacanthos, H. tuberosus, P. serotina, Ph. virginiana, $S$. sclarea, S. nigrum and U. urens) presented in Table 2, all the plants were associated and feed by M. pruinosa also in Austria and the degree of infestation was similar too to those of showed in Tables 3-7. Preda and Skolka (2011) sampled 37 places (parks with seminatural vegetation) along the Black See in Romania. Despite the geographical position difference of Hungary and Romania, the most frequent plants on which $M$. pruinosa commonly occurred in the Romanian study were similar to those of this study: A. negundo, A, platanoides, A. altissima, E. japonica, Fraxinus sp., P. cerasifera, Ph. coronarius, R. pseudoacacia and $S$. $x$ vanhuttei). Another study (Grozea et al., 2015) conducted in the western counties of Romania, showed an even better match with the present data: A. campestre, A. negundo, A. platanoides, B. sempervirens, C. sanguinea, H. lupulus, J. regia, M. domestica, P. domestica, Ph. coronarius, R. pseudoacacia, R. damascena, S. x vanhuttei, U. campestris and $V$. vinifera were common hostplants in both countries. This list corresponds also to the Austrian observations mentioned above.

Tables 8-11 present - depending on the habitats' character - a functional classification (ornamental plants, trees/shrubs, fruit plants, weeds and feral plants) of the host plant families observed at the various habitats. In seminatural habitats (hedge and tree row) the trees and shrubs predominated but in areas of agricultural use (backside gardens, orchard and vineyard) ornamental plants and not cultivated deciduous trees were in the majority. Feral plants could be found almost at each site. Some of them such as $S$. canadensis or P. serotina, are invasive alien species of North American origin. The number of plants native to America, some of them introduced to Europe as ornamental plants or trees of agricultural or forestry importance is 10: A. artemisiifolia, A. negundo, C. occidentalis, C. bipannatus, G. triacanthos, $H$. tuberosus, P. serotina, Ph. virginiana, $R$. pseudoacacia, S. canadensis. Considering the frequency of host plants recorded and their degree of infestation of the planthopper, Asteraceae, Cannabaceae, Fabaceae, Juglandaceae, Lamiaceae, Rosaceae and Sapindaceae were the significant families. At least two host plants of American origin were found at each site not respecting the alfalfa field and the nettle stand.

Abiotic conditions like temperature, precipitation, moisture of air and soil play an important role in development, reproduction, survival and spread of CFP. In dry regions its distribution is limited (Strauss, 2009). This can be observed during surveys because in hot and dry conditions e.g. on the top of shoots and places exposed to direct sunshine and high temperature the $M$. pruinosa populations were very scarce or nil. Thus, one could often experience that the host plant preference was influenced by the abiotic conditions of the habitat and the plant density.

A number of papers mentioned the honeydew production of M. pruinosa (Souliotis et al., 2008; Kahrer et al., 2009; Strauss, 2009). Kahrer et al. (2009) stressed that a high quantity of honeydew was observed on Acer spp., Malus sp., Parthenocissus quinquefolia and Clematis vitalba in August. In order to study the honeybee's response to $M$. pruinosa honeydew, hives were placed adjacent to infested areas but no honeybee flight was detected. As to present investigations no honeydew of $M$. pruinosa has been perceived.

M. pruinosa density approached damage level on roses and raspberries in the Máriabesnyő backside garden in 2015. On raspberries the shoots and leaves were so heavily attacked that their development was hampered while on roses the presence of CFP was the damage. The three applications of Vectaphid A EC were efficient to stop the population increase and prevented the further damage. How is possible to manage potential outbreaks of CFP a more long-lasting or radical way? In Great Britain and Bohemia chemical eradication was successful (C. Malumphy and P. Lauterer, personnel communication in Strauss, 2009). Perhaps, it was due to the generally colder and more humid climate of both countries which did not favoure the development and reproduction of the pest. Thus, this eradication with pesticides in Hungary cannot be a right answer. In Austria there was a mass outbreak in Vienna in 2003 and the pest continued spreading and was found also in Graz. According to the risk analysis of Strauss (2010) mainly organic orchards and vineyards in Burgerland, Lower Austria and Styria are threatened by $M$. pruinosa. She proposed inspection of trade and trade pathways of trees and ornamentals, parking sites and gardens along transport routes, pesticide application and the introduction of Neodryinus typhlocybae. What can we do in Hungary? Our climatic conditions are more favourable for the planthopper than those in Austria. Our facilities (personnel or material) are limited. The spread of M. pruinosa is not estimated and known. The only efficient and environmental friendly control opportunity might be the introduction of 
natural enemies, in this case Neodryinus typhlocybae already established in Italy, France, Switzerland, Slovenia and Croatia (Tommasini et al., 1998; Ciglar et al., 1998; Malausa, 1999; Jermini et al., 2000 in Strauss, 2009; Žeźlina et al., 2001). In 2007, the parasitoid has been released in Greece, the Netherlands and Spain (A. Sala, personal communication in Strauss, 2009). N. typhlocybae was tested for not target organisms in Austria and it is likely that its host range is restricted to Flatidae, of which merely CFP occurs in Austria (Strauss, 2009).

\section{CONCLUSIONS}

Host plant diversity and preference of M. pruinosa were detected from 2011 to 2015 at Gödöllö and its countryside. The pest could be found at each habitat sampled but the hedge, the tree row and the gardens as well as the orchard and vineyard proved to be the most infested. Frequency and population density of CFP were considerable on Asteraceae, Cannabaceae, Fabaceae, Juglandaceae, Lamiaceae, Rosaceae and Sapindaceae. Vegetation could be functionally classified as ornamental plants, trees, fruit plants, weeds and feral plants. Feral plants - some of them also invasive alien species - were found at each habitat. Plant species native to America were among them the most populated. This fact obviously might help the establishment and spread of $M$. pruinosa. Regarding the neglected hedgerows, the predominance of abandoned gardens, orchards and vineyards, there are excellent conditions (high plant diversity with many American plant species) for the quick and longlasting establishment of $M$. pruinosa. The length of hedges which run often parallel with the railway line can be more than several hundred km only in Pest county. This means that the hedge can be a huge reservoir of $M$. pruinosa. These hedges have enormous beneficial importance as resources for firewood, medicinal plants, fruits, berries, mushrooms, bee pastures; structures like ecological networks, corridors and barriers, shelter for protected plant and animal species, and natural enemies. The general management of these hedges is cutting the trees in every five or 10 years to gain some heating material and making a better view. It is a chance that there is neither money, nor intension to apply chemical insecticides in these structures. This means that in case of introduced invasive pests without natural enemies, there is quite a high risk for establishing and spreading in such a highly suitable, new environment especially when many formerly established feral plants make easier this process. Although, the population density of the pest was considerable on many hostplants, economic damage or yield losses could not be assessed. Economic or significant damage was observed only on roses, raspberries and stinging nettle (there are recognised stinging nettle varieties in Germany and Finland; Dreyer, 1999). This highlights that CFP outbreaks may cause under favourable ecological circumstances considerable quality and yield loss. The most favourable and long-ranging control opportunity would be the introduction of the natural enemy, Neodryinus typhlocybae already introduced in many European countries. Concerning the environmental and climatic conditions as well as the crop protection opportunities of the country it is dubious that continual inspections or verifications could help not to mention the chemical eradication.

\section{REFERENCES}

Bagnoli, B. and Lucchi, A. (2000): Dannosità e misure di controllo integrato. In: A. Lucchi, (ed.). La Metcalfa negli ecosistemi italiani. ARSIA. Regione Toscana, Italy. pp. 65-88.

Bozsik A. (2012): Mass occurrence of the citrus flatid planthopper (Metcalfa pruinosa (Say, 1830)) (Hemiptera: Flatidae) in an agricultural hedgerow at Gödöllő (Hungary). Journal of Agricultural Sciences, Debrecen, 50 (Supplement), 115-118.

Ciglar, I., Barić, B., Žužic, I. (1998): Biological control of Metcalfa pruinosa by the introduction of Neodryinus typhlocybae (Ashmead) (Hymenoptera: Dryinidae) to Croatia. Fragmenta Phytomedica et Herbologica, 26(1/2), 95-99.

Della Giustina, W. (1987): Metcalfa pruinosa (Say 1830), nouveaute pour la faune de France (Hom. Flatidae). Bulletin de la Societé Entomologique de France 191(3-4), 89-92.

DAISIE Metcalfa pruinosa factsheet. Dekivering alian invasive species inventories for Europe. http://www.europe-aliens.org/ Accessed 26 September 2012

Dreyer, J. (1999): Die Fasernessel als nachwachsender Rohstoff. Leistungsprüfung von Fasernesseln (Urtica dioica L., Grosse Brennessel) unter besonderer Berücksichtigung der phänotypischen Differenzierung anbauwürdiger Klone. Verlag Dr. Kovač, Hamburg, pp. 206.

Grozea, I., Gogan, A., Virteiu, A.M., Grozea, A., Stef, R., Molnar, L., Carabet, A. and Dinnesen, S. (2011): Metcalfa pruinosa Say (Insecta: Homoptera: Flatidae): a New pest in Romania. African Journal of Agricultural Research 6 (27), 5870-5877.

Grozea, I., Gogan, A., Vlad,M., Virteiou, A.M., Stef, R., Carabet, A., Damianov, S., Florian, T. (2015): A new problem for Western Romania: Metcalfa pruinosa emiptera: Flatidae. BulletinUASVM Horticulture, 72 (1), 74-80.

Kahrer, A, Moosbeckhofer, R. (2003): Ein neuer Schädling -Metcalfa pruinosa - in Österreich eingeschleppt. Bienenvater 10, 16.

Kahrer, A., Strauss, G., Stolz, M., Moosbeckhofer, R. 2009 Beobachtung zur Faunistik and Biologie der vor kurzem nach Österreich eingeschleppten Bläulingszikade (Metcalfa pruinosa). Beiträge zur Entomofaunistik, 10,17-30.

Lauterer, P. (2002): Citrus flatid planthopper, Metcalfa pruinosa (Hemiptera: Flatidae), a new pest of ornamental horticulture in the Czech Republic. Plant Protection Science 38(4): 145-148.

Malausa, J.C. (1999): Un espoir face aux pullulations de Metcalfa pruinosa. Introduction en France de Neodryinus typhlocybae, parasite larvaire de cette „cicadelle”. Phytoma, 512,37-40.

Mead, F.W. (1969): Citrus flatid planthopper, Metcalfa pruinosa (Say) Homoptera: Flatidae. Florida Department of Agriculture, Division of Plant Industry, Entomology Circular No. 85. 1-2. 
Mihajlović, Lj. (2007): Metcalfa pruinosa (Say) (Homoptera: Auchenorrhyncha) a new harmful species for entomofauna of Serbia. Glacnik Sumarskog Fakulteta, Beograd, 95, 127-134.

Moosbeckhofer, R., Heigl, H., Kahrer, A., Strauss, G., Stolz, M. (2009): Untersuchungen zum Auftreten der Bläulingszikade Metcalfa pruinosa (Say 1830; Hemiptera, Flatidae), einer in Österreich neuen Honigtauerzeugerin, und die möglichen Auswirkungen auf die Bienenzucht (Investigations on the incidence of Metcalfa pruinosa (Say 1830; Hemiptera, Flatidae), a new honeydew producing insect in Austria, and its possible implication on beekeeping). pp. 39. http://www.ages.at/fileadmin/_migrated/content_uploads/ Abschlussbericht_Metcalfa_Pruinosa_Bienenzucht_SRL.pdf

Orosz A. és Dér Zs. (2004): Idejében szólunk a Metcalfa pruinosa (Say, 1830) kabóca esetleges megjelenéséröl. Növényvédelem 40 (3), 137-141.

Preda, Ch., Skolka, M. (2011): Range expansion of Metcalfa pruinosa (Homoptera: Fulgoroidea) in Southeastern Europe. Ecologia Balcanica 3(1), 79-87.

Rebek, E.J. (2009): Pest du Jour: Flatid Planthoppers. Pest alerts, Oklahoma State University 8(16), 2. http://entoplp.okstate.edu/Pddl/

Souliotis,C, Papanikolaou, N.E., Papachristos, D. and Fatouros, N. (2008): Host plants of the planthopper Metcalfa pruinosa (Say) (Hemiptera: Flatidae) and bservations on its phenology in Greece. Hellenic Plant Protection Journal 1, 39-41.

Strauss, G. (2009): Host range testing of the nearctic beneficial parasitoid Neodriynus typhlocybae. Biocontrol 54, 163-171.

Strauss, G. (2010): Pest risk analysis of Metcalfa pruinosa in Austria. Journal of Pest Science 83(4), 381-390.Tommasini, M.G., Mosti, M., Dradi, D., Girolami, V. (1998): Lotta biologica contro Metcalfa pruinosa con Neodryinus typhlocybae: prime esperienze sull'acclimatazione del parassitoide in Emilia-Romagna. Inf. Fitopatol. 48(12), 51-54.

Zangheri, S. and P. Donadini. (1980): Comparsa nel Veneto di un omottero neartico: Metcalfa pruinosa Say (Homoptera, Flatidae). Redia 63, 301-305.

Žeźlina, I., Milevoj, L. \& Girolami, V., (2001): Wasp Neodrynus typhlocybae Ashmead - succesuful predator and parasitoid for reducing the population of flatid planthopper (Metcalfa pruinosa Say) also in Slovenia. - Zbornik Bioteh. Fak. Univ Ljubljana. Kmet. 77(2), 215-225. 